\title{
Diagnóstico das Condições de Saúde de uma Comunidade Garimpeira na Região do Rio Tapajós, Itaituba, Pará, Brasil, 1992
}

\author{
Diagnosis of Health Conditions in a Pan-Mining Community in the Tapajós \\ River Basin, Itaituba, Pará, Brazil, 1992
}

\author{
Elisabeth de O. Santos ${ }^{1}$; Edvaldo Carlos B. Loureiro' ; Iracina Maura de Jesus ${ }^{\text {; }}$; \\ Edilson Brabo'; Rita do S. U. da Silva'; Manoel do C. P. Soares'; \\ Volney de M. Câmara²; Maria dos R. S. de Souza' \& Fernando Branches ${ }^{3}$
}

SANTOS, E. O.; LOUREIRO, E. C. B.; JESUS, I. M.; BRABO, E.; SILVA, R. S. U.; SOARES, M. C. P.; CÂMARA, V. M.; SOUZA, M. R. S. \& BRANCHES, F. Diagnosis of Health Conditions in a Pan-Mining Community in the Tapajós River Basin, Itaituba, Pará, Brazil, 1992. Cad. Saúde Públ., Rio de Janeiro, 11 (2): 212-225, Apr/Jun, 1995.

The purpose of this article is to describe health conditions in a pan-mining community, in particular the interaction of mercury contamination with other health problems. This study was carried out in a panning mine located on Rato Creek, a tributary of the Tapajós River Basin in the municipality of Itaituba, State of Pará The study population consisted of 223 individuals. An epidemiological questionnaire was applied, medical treatment was given, and biological specimens were collected. Testing of fecal samples showed that $96.1 \%$ of the individuals had parasites and $66.4 \%$ were anemic. Prevalence of hepatitis B virus was $85.0 \%$, and 11 individuals $(6.0 \%)$ were virus carriers (HBsAg positive). Of 186 patients tested for malaria, 65 individuals (35.0\%) had the infection, of whom 34 (52.3\%) were asymptomatic. Prevalence of syphillis was 41.6\%. Urine mercury levels were measured in 173 individuals. Some $16(9.2 \%)$ of those tested had mercury levels between 10 and $19 \mathrm{ug} / \mathrm{l}$, while 9 patients (5.2\%) had levels above $20 \mathrm{ug} / \mathrm{l}$. An overview of general health conditions showed a combination of problems in individuals, who lacked adequate diagnosis, in addition to a high level of self-medication, given the absence of health facilities. In addition, these individuals are threatened by critical levels of mercury contamination. In conclusion, this article gives a general overview of health conditions in these pan-mining communities, demonstrating the multicausal nature of health conditions in the Amazon region, a fact which should be considered when planning appropriate strategies for clinical treatment.

Key words: Environmental Health; Worker's Health; Pan Mining; Mercury

\footnotetext{
'Laboratórios de Ecologia Humana e Meio Ambiente, Instituto Evandro Chagas. Av. Almirante Barroso, 492, Belém, PA, 66090-000, Brasil.

${ }^{2}$ Núcleo de Estudos de Saúde Coletiva, Universidade Federal do Rio de Janeiro. Av. Brigadeiro Trompowski, Hospital Universitário, 5o andar, Rio de Janeiro, RJ, 21941-590, Brasil.
}

${ }^{3}$ Av. São Sebastião, 1219, Santarém, PA, 68040-040, Brasil.

\section{INTRODUÇÃO}

O crescimento acentuado da produção brasileira de ouro nos últimos quinze anos tem colaborado para uma alteração marcante do padrão de morbidade na região amazônica.

Segundo cálculos estimados por Félix (1987) e pela Secretaria de Estado de Indústria Comércio e Mineração do Pará (Seicom, 1992), a produção de ouro no Brasil que em 1972 era de 9,6 toneladas, cresceu continuamente até o início da década de 1980, quando alcançou 37 tonela- 
das. Em 1983, ela sobe dramaticamente para 70 toneladas, quase o dobro, e no fim da década de 80 chega perto de 111 toneladas, o triplo.

Dada a elevada contribuição do ouro do garimpo para a produção total, bem como a notória dependência da garimpagem em relação à mão-de-obra, pode-se desde logo concluir que o crescimento do produto implicou intensa mobilização de contingentes humanos. É difícil precisar o número de garimpeiros existentes no Brasil. A única fonte oficial que existe é um cadastro realizado pelo Departamento Nacional de Produção Mineral em 1990 (DNPM, 1990). Este cadastro mostrou um número provavelmente subestimado de 419.920 trabalhadores (Tabela 1), distribuídos principalmente no Estado do Pará, sendo seguido por Mato Grosso, Rondônia e Goiás.

TABELA 1. Distribuição da População Garimpeira por Estados da União, Brasil, 1990

\begin{tabular}{lrr}
\hline \hline $\begin{array}{l}\text { Estados } \\
\text { Garimpeiros }\end{array}$ & $\mathrm{N}^{\mathrm{o}}$ & $\%$ \\
\hline Pará & 221.500 & 52,7 \\
Mato Grosso & 100.700 & 24,0 \\
Rondônia & 22.200 & 5,3 \\
Goiás & 21.350 & 5,1 \\
Outros Estados & 54.170 & 12,9 \\
\hline Total & 419.920 & 100 \\
\hline \hline
\end{tabular}

Fonte: Cadastro do DNPM (1990).

Estudos específicos sobre a distribuição do mercúrio usado na atividade garimpeira nos diferentes compartimentos ambientais, água, sedimento, ar, biota, têm sido desenvolvidos na região amazônica, contribuindo para o melhor entendimento dos mecanismos de dispersão deste metal no ambiente (Martinelli et al., 1988; Pfeiffer et al., 1989; Ramos \& Costa, 1991; Hacon, 1991 e Brabo, 1992). A prioridade que vem sendo dada ao mercúrio nesses estudos se justifica quando calculamos as quantidades desse agente lançadas no meio-ambiente nos últimos quinze anos, a partir da produção declarada de ouro para o mesmo período, e compara- mos com a gravidade do que pode estar acontecendo em natureza com o risco potencial de biomagnificação do mesmo, através da cadeia biológica (Brabo, 1992).

A produção científica sobre os efeitos para a saúde humana em decorrência desse tipo de atividade econômica, entre os garimpeiros, na maioria das vezes se restringe aos riscos causados pela exposição ao mercúrio utilizado na concentração do ouro através de amalgamação e posterior queima deste amálgama. Entretanto, Santos (1993) ressalta que a avaliação dos problemas de morbidade e mortalidade da região amazônica, mesmo quando se trata de área garimpeira, não pode ficar restrita exclusivamente àqueles causados pela intoxicação por mercúrio, os quais devem ser investigados paralelamente às demais causas envolvidas no processo saúde/doença.

Uma análise da rotina de trabalho, das relações de trabalho e das condições de vida nas áreas de garimpo realizada pelo Centro Panamericano de Ecologia Humana e Saúde da Organização Panamericana da Saúde (Câmara \& Corey, 1992) apontou alguns riscos por agentes químicos, físicos, mecânicos, biológicos, ergonômicos e psicossociais que poderiam estar associados à presença de vários efeitos adversos à saúde dos garimpeiros, com destaque para a intoxicação por mercúrio, violência, a malária, as doenças sexualmente transmissíveis, e a dependência em relação a diversos tipos de drogas.

Sendo assim, a investigação da saúde humana sob o ponto de vista das agressões provocadas ao meio ambiente na Amazônia, inclui obrigatoriamente a necessidade de se considerarem as diferentes variáveis que agem simultaneamente sobre os organismos individuais, resultando freqüentemente em sinais e sintomas semelhantes. A avaliação desta relação de causa e efeito não é simples nem direta, motivo pelo qual as investigações devem contemplar informações clínicas, epidemiológicas e provas laboratoriais, que facilitem o entendimento da realidade que se quer conhecer.

O presente estudo faz parte de um projeto maior que tem como objetivo avaliar o estado geral de saúde em diferentes compartimentos epidemiológicos na Amazônia, inclusive no que diz respeito à intoxicação mercurial. $\mathrm{O}$ trabalho 
iniciou com a aplicação de uma ficha epidemiológica, exame clínico geral, exame específico para a clínica de mercúrio, coleta de espécimes e tratamento dos agravos encontrados. No momento apresentamos alguns dados necessários para compor uma visão geral das condições de saúde de uma comunidade garimpeira no Estado do Pará, procurando estabelecer relações entre o estado geral dos indivíduos e as condições de vida e de trabalho predominantes nesse agrupamento humano.

\section{MATERIAIS E MÉTODOS}

\section{Área e População}

A região do Tapajós tem como principal atividade econômica a extração mineral, sobretudo de ouro, cujas primeiras ocorrências registradas remontam ao século XVIII (Fernandes \& Portela, 1991). A área garimpeira investigada localiza-se na bacia hidrográfica do rio Tapajós, no igarapé do Rato, tendo como ponto central a pista de pouso conhecida como "Piririma", abrangendo os vários núcleos de garimpagem dispostos ao longo de suas margens ou proximidades, bem como as balsas que atuam na área revolvendo o leito do igarapé e um vilarejo próximo da pista, chamado de "Currutela do João Leite", situados num raio de cerca de $35 \mathrm{~km}$ da pista de pouso.

A população estudada compreendeu as pessoas moradoras na área garimpeira, incluindo garimpeiros e não garimpeiros, tais como cozinheiros, prostitutas, crianças, pessoas sem ocupação e outros. O número de indivíduos foi calculado - na falta de dados oficiais - a partir de informações obtidas com os proprietários dos garimpos da região, somadas a observações feitas no local durante uma visita de reconhecimento prévio, e estimado em cerca de 300 pessoas no total, dos quais posteriormente se verificou que 55\% eram provenientes de outros estados. Considerando a escassez de dados e a irregularidade das fontes de informação, optou-se por trabalhar com o máximo possível de indivíduos residentes na área, para tentar cobrir a totalidade da população estimada.

\section{PROCEDIMENTOS DE CAMPO}

a. Aplicação individual de um questionário epidemiológico, incluindo informações sobre identificação, condições de vida, história ocupacional, hábitos alimentares, morbidade; avaliação clinica dos casos, tratamento quando necessário, e um documento de consentimento.

b. Montagem de um laboratório de campo para coleta, identificação, separação e conservação de espécimes biológicos.

c. Atendimento médico e coleta de espécimes biológicos na população geral, com ou sem queixa clínica no momento.

Entre todos os entrevistados foram coletados os espécimes abaixo relacionados, segundo as respectivas finalidades:

a. Urina - exame de rotina e quantificação de mercúrio total.

b. Sangue/soro - diagnóstico de malária, hemograma, algumas dosagens bioquímicas, além de sorologia para Malária, Hepatite B e Sífilis.

c. Fezes - parasitoscopia direta.

As amostras de urina para dosagem de mercúrio foram coletadas em dois recipientes plásticos com capacidade de $25 \mathrm{ml}$ cada, hermeticamente fechados, a partir da primeira micção, e conservados a $-20^{\circ} \mathrm{C}$ até o momento da análise, de acordo com Campos \& Pivetta (1993). As determinações de mercúrio total foram realizadas pelo Laboratório de Toxicologia do Instituto Evandro Chagas, e pelo Laboratório de Radio-isótopos do Instituto Carlos Chagas da Universidade Federal do Rio de Janeiro (UFRJ), por absorção atômica através da técnica de geração de vapor a frio. Os demais espécimes foram conservados e transportados em nitrogênio líquido.

O diagnóstico laboratorial dos casos de malária foi feito através da leitura da lâmina de gota espessa. A pesquisa sorológica para a sífilis em soros de 185 pacientes foi realizada pelo método de micro-hemaglutinação, utilizando o "kit" Hemapallidum da BIOLAB. As demais pesquisas sorológicas foram feitas pela técnica imunoenzimático de ELISA. 
A colimetria da água dos dois poços e a do igarapé do Rato, para a obtenção do Número Mais Provável (NMP/100ml) foi realizada pelo método dos tubos múltiplos.

\section{RESULTADOS}

Os resultados aqui apresentados referem-se àqueles mais significativos dentre os que foram obtidos através da análise das fichas epidemiológicas e/ou de determinações laboratoriais.

\section{Identificação da População}

Foram entrevistados 223 indivíduos garimpeiros ou não (74,3\% da população estimada), distribuídos por faixa etária e sexo (Tabela 2).
A composição da amostra populacional por sexo revela $67 \%$ (149) de indivíduos do sexo masculino e $33 \%$ (74) do feminino. As idades variaram entre 6 meses e 59 anos, com a maior concentração de pessoas entre 20 e 40 anos $(71,3 \%)$, na chamada faixa produtiva. Na população de garimpeiros o sexo masculino predominou sobre o feminino com $98,3 \%$, enquanto que na população não garimpeira, 70,6\% dos indivíduos pertenciam ao sexo feminino.

A análise dos dados demonstrou que a naturalidade paraense (44\%) predominou sobre a de outros Estados, quando se considera a população geral, ficando o Maranhão em segundo lugar com $35 \%$. Essa ordem sofre inversão quando se considera apenas o grupo garimpeiro, onde predominam os maranhenses com $47,0 \%$.

TABELA 2. Distribuição da População do Garimpo do Rato Segundo Faixa Etária, Sexo e Atividade Ocupacional. Itaituba, Pará, Setembro, 1992

\begin{tabular}{lrrrrrrrrrrrr}
\hline \hline Faixa & \multicolumn{4}{c}{ Garimpeiro } & \multicolumn{4}{c}{ Não Garimpeiro } & \multicolumn{3}{c}{ População Geral } \\
\cline { 2 - 11 } Etária & M & \multicolumn{1}{c}{ F } & \multicolumn{1}{c}{ T } & $\%$ & M & \multicolumn{1}{c}{ F } & T & 96 & M & F & T & 96 \\
\hline $0-4$ & - & - & - & - & 6 & 3 & 9 & 8,8 & 6 & 3 & 9 & 4,0 \\
$5-9$ & - & - & - & - & - & 4 & 4 & 3,9 & - & 4 & 4 & 1,8 \\
$10-14$ & 1 & - & 1 & 1,0 & 6 & 2 & 8 & 7,8 & 7 & 2 & 9 & 4,0 \\
$15-19$ & 7 & - & 7 & 5,8 & - & 5 & 5 & 4,9 & 7 & 5 & 12 & 5,4 \\
$20-29$ & 58 & 1 & 59 & 48,8 & 8 & 33 & 41 & 40,2 & 66 & 34 & 100 & 48,8 \\
$30-39$ & 32 & 1 & 33 & 27,2 & 5 & 21 & 26 & 25,5 & 37 & 22 & 59 & 26,5 \\
$40-49$ & 13 & - & 13 & 10,7 & 2 & 4 & 6 & 5,9 & 15 & 4 & 19 & 8,5 \\
$50-59$ & 6 & - & 6 & 4,9 & 3 & - & 3 & 2,9 & 9 & - & 9 & 4,0 \\
Ignorada & 2 & - & 2 & 1,6 & - & - & - & - & 2 & - & 2 & 1,0 \\
\hline Total & 119 & 2 & 121 & 100 & 30 & 72 & 102 & 100 & 149 & 74 & 223 & 100 \\
\hline \hline
\end{tabular}

Ainda do inquérito, $58,0 \%$ dos entrevistados identificaram-se como solteiros e $40,0 \%$ como casados ou amasiados. Entretanto, apenas $27,0 \%$ preferem informar que vivem com suas famílias, percentual que cai para $10,0 \%$ no grupo garimpeiro.

Quanto à formação escolar, mais de $64,0 \%$ referiram algum nível de instrução básica sem completar o $1^{\circ}$ grau; $24,0 \%$ declararam-se analfabetos ou apenas capazes de escrever o próprio nome e somente $3,0 \%$ concluíram o $1^{\circ}$ grau. As crianças, sem escola para freqüentar, reiniciam o padrão, transpondo para o futuro a mesma situação de carência educacional de hoje.
Quanto à distribuição dos entrevistados segundo a atividade ocupacional prévia, comércio e agricultura alcançaram 23,0\% cada um; garimpo $21,0 \%$; construção civil $7,0 \%$; prostituição $2,0 \%$ e outros $25,0 \%$. É interessante observar que a grande maioria dessa população $(74,0 \%)$ exercia essas atividades ocupacionais prévias, em áreas não garimpeiras.

\section{Hábitos Pessoais}

Tanto na população geral quanto no grupo garimpeiro 70,0\% fuma ou já fumou por muito tempo. A ingestão rotineira, diária ou semanal, atual ou não, de bebidas alcoólicas atinge $81,0 \%$ dos 
entrevistados. Entretanto, quando se considera o uso de drogas ilegais, as informações são mais escassas, e apenas $10,0 \%$ relataram contato com as mesmas em alguma oportunidade, sendo a maconha e a cocaína as mais citadas.

\section{Nutrição}

A dieta alimentar básica do grupo estudado consta de carne, peixe, feijão, farinha de mandioca e de milho, arroz, poucos legumes e praticamente nenhuma fruta. $\mathrm{O}$ consumo de peixe foi citado por mais de $80,0 \%$ dos entrevistados, procedendo o pescado principalmente de Itaituba $(62,0 \%)$, ou do próprio igarapé do Rato $(36,0 \%)$, sendo que, em qualquer dos casos, o pescado procede da bacia do Tapajós sobre a qual pairam suspeitas de poluição por mercúrio. A freqüência média de ingestão deste alimento é de uma a duas refeições por semana.

\section{Saneamento e Parasitoses}

O abastecimento de água da maioria da população é proveniente de poços abertos $(47,5 \%)$, e chega encanada e sem nenhum tipo de tratamento em apenas 33,0\% das moradias, principalmente nas "corrutelas". A utilização direta da água do rio foi referida por $16,0 \%$ dos indivíduos, e cerca de 4,0\% consomem água de cisternas ou outras coleções hídricas. A população como um todo utiliza a água sem qualquer tipo de tratamento.

A colimetria feita nas 2 cisternas superficiais da pista de pouso do Piririma e em 4 amostras colhidas ao longo do igarapé do Rato, próximo às margens, demonstrou a presença abundante de coliformes fecais, com variação do Número Mais Provável (NMP/100ml) de 220 a 300 por $100 \mathrm{ml}$. A normalidade para uso no abastecimento é - segundo a Resolução no 20 de 18 de junho de 1986 do Conselho Nacional do Meio Ambiente (Conama, 1986) - ausência de coliformes totais em qualquer amostra.

Sobre o destino dos dejetos, $58,5 \%$ são depositados a céu aberto e somente $41,5 \%$ da população dispõem de local específico através de fossa negra $(32,4 \%)$ ou fossa seca $(9,1 \%)$. Em $80 \%$ dos casos, o lixo geral é despejado às margens da mata e, pelo menos $10,0 \%$ das pessoas lançam-no diretamente no rio.
Os dados de morbidade por doenças do aparelho digestivo revelam que $71 \%$ das pessoas entrevistadas possuem problemas intestinais e, destes, 90\% relataram ocorrências em um período inferior a um ano, antes da coleta dos dados.

A coproscopia realizada em 154 indivíduos da população do garimpo do Rato, revelou 96.1 $\%$ de parasitados. Os parasitos encontrados segundo a percentagem de indivíduos infectados foram: Ancilostoma duodenale 94, (61,0\%), Ascaris lumbricoides 73, (47,0\%), Entamoeba coli 30, (19,5\%), Entamoeba histolytica 21, (13,6\%), Endolimax nana 19, (12,3\%), Iodamoeba butschillii 12, (7,8\%), Giardia lamblia 8, (5,2\%), Trichocephalus trichiurus 5, (3,2\%), Strongiloides stercoralis $1,(0,6 \%)$ e Enterobius vermiculares $1,(0,6 \%)$.

Em 120 pacientes portadores de parasitose intestinal foi feita a hematimetria, que revelou a presença de anemia em 57,5\%, percentual representado pelos indivíduos que apresentaram valores de hemoglobina abaixo da normalidade, considerando-se como limites para o sexo feminino 11,5, e para o masculino 12,5 unidades, segundo o método HBCN (Todd \& Sansord e Davidsohn, 1982). Toda a medicação indicada para tratamento de parasitoses intestinais levada para campo foi usada na população do garimpo do Rato.

\section{Morbidade por Malária}

Praticamente toda a população estudada $(94,0 \%)$ referiu episódios de malária nos últimos dez anos. Esses números entre os garimpeiros alcançaram $98,0 \%$ - de um total de 121 pessoas, apenas 2 disseram nunca terem tido esse agravo. Além disso, 69,0\% da população referem ter tido a doença no decorrer do último ano.

$\mathrm{Na}$ distribuição dos entrevistados segundo os episódios de malária, observa-se que $29,0 \%$ já haviam sofrido de um a dois episódios; $26,0 \%$ mais de dois episódios; $23,0 \%$ de três a cinco; e $22,0 \%$ de seis a doze episódios. É importante citar também que $97 \%$ sofreram o último episódio no garimpo.

O nível de procedimentos preventivos citados pelos entrevistados é baixo (7\%), sendo que a maioria o faz através de medicamentos. Entretanto, $19 \%$ desta população referiram a utiliza- 
ção de mosquiteiro sem considerá-lo como medida preventiva contra malária.

O resultado da leitura das lâminas colhidas, para exame de malária, no campo, em 186 indivíduos sintomáticos e assintomáticos, analisado por sexo e atividade ocupacional revela que $35,0 \%$ (65) das pessoas testadas estavam com a doença. Na Tabela 3 encontram-se relatados os resultados dos 65 pacientes positivos para malária, por sexo e situação ocupacional, tendo como referência o estado clínico no momento da coleta, isto é, se era sintomático ou assintomático. Dentre as 65 lâminas positivas, $34(52,3 \%)$ foram colhidas de pacientes sem queixa clínica. No grupo garimpeiro, dentre os 32 examinados, 19 (59.4\%) estavam assintomáticos e $13(40.6 \%)$ relataram sintomas da doença.

\section{Anemia}

Entre os 148 indivíduos dos quais foram obtidos resultados do hemograma que permitiram a avaliação de alterações da hematimetria, em $66,4 \%$ dos casos o paciente revelava níveis de hemoglobina abaixo da normalidade, caracterizando o estado anêmico. Quando coincidiu termos resultados da hematimetria em pacientes positivos para malária e parasitose intestinal, $61,2 \%$ estavam anêmicos. Considerando só a presença da malária, os pacientes anêmicos constituem $64,2 \%$ da amostra analisada.

\section{Morbidade por Mercúrio Metálico}

Diversas queixas clínicas referidas no inquérito oferecem informações sobre possível mor-

TABELA 3. Resultados dos 65 Pacientes Positivos para Malária, Segundo Sexo, Situação Ocupacional e Presença de Sintoma Malárico. Garimpo do Rato. Itaituba, Pará, Setembro, 1992

\begin{tabular}{|c|c|c|c|c|c|}
\hline \multirow[b]{3}{*}{ Ocupação } & \multicolumn{4}{|c|}{ Sexo } & \multirow{3}{*}{ Total } \\
\hline & \multicolumn{2}{|c|}{ Masculino } & \multicolumn{2}{|c|}{ Feminino } & \\
\hline & Sintomático & Assintomático & Sintomático & Assintomático & \\
\hline Garimpeiro & 13 & 19 & - & - & 32 \\
\hline Prostitutas & $-*$ & $-*$ & 5 & 1 & 6 \\
\hline Cozinheiros & - & - & 3 & 3 & 6 \\
\hline Outras & 4 & 3 & 4 & 3 & 14 \\
\hline Sem Ocupação & - & 2 & 2 & 3 & 7 \\
\hline Total & 17 & 24 & 14 & 10 & 65 \\
\hline
\end{tabular}

* Não foi possível obter dados sobre prostituição masculina.

bidade existente na comunidade relacionada coma presença do mercúrio no meio ambiente, não só daquele proveniente da queima do amálgama, como também através do contacto direto com o mercúrio metálico durante o trabalho de extração do ouro. Dos garimpeiros entrevistados, $11 \%$ referiram problemas de saúde que, segundo eles, teriam sido causados por exposição ao mercúrio. Destes, $43 \%$ assinalaram sinais ou sintomas na pele, $13 \%$ astenia, e $43 \%$ outros sintomas. Dos entrevistados que relataram estes problemas de saúde, $22 \%$ referiram absenteísmo do trabalho por esta causa. A Tabela 4 relaciona os principais sintomas passíveis de serem associados com exposição ao mercúrio metálico, divididos pelo tempo de exposição ao $\mathrm{Hg}$, com destaque para irritabilidade, nervosismo, palpitação, e perda de memória (Yoshida \& Yamamura, 1982), que demonstraram correlação positiva com a hipótese de intoxicação por esse metal. O tempo predominante de exposição ao mercúrio foi de 6 a 10 anos $(43,0 \%)$, seguido de 1 a 5 anos $(34,0 \%)$, mais de 10 anos $(20,0 \%)$ e menos de 1 ano (30\%). 
TABELA 4. Principais Sintomas Referidos, Passíveis de Associação à Exposição ao Mercúrio Metálico, Segundo Tempo de Permanência. Garimpo do Rato, Itaituba, PA, 1992

\begin{tabular}{lccccccc}
\hline \hline & \multicolumn{5}{c}{ Tempo de Exposição } & & \\
\cline { 2 - 5 } & \multicolumn{2}{c}{ Até 5 } & Anos & Mais que & Anos & & \\
\cline { 2 - 5 } Sintomatologia Referida & Sim & Não & Sim & Não & $X^{2}$ & P \\
\hline Depressão & 10 & 107 & 15 & 91 & 1,75 & 0,18 \\
Insônia & 26 & 91 & 32 & 74 & 1,83 & 0,17 \\
Irritabilidade & 25 & 41 & 92 & 65 & 8,00 & 0,004 \\
Nervosismo & 45 & 72 & 65 & 41 & 11,63 & 0,0006 \\
Palpitação & 19 & 98 & 40 & 66 & 13,21 & 0,0002 \\
Perda de Memória & 38 & 79 & 49 & 57 & 4,42 & 0,035 \\
\hline \hline
\end{tabular}

Os resultados das dosagens de mercúrio em urina, coletada de 173 indivíduos dentre os componentes da amostra (Tabela 5), revelaram $148(85,5 \%)$ desses pacientes nos quais as quantidades de mercúrio estavam inferiores à faixa de $10 \mathrm{ug} / \mathrm{l}$, indicando níveis pouco significativos de exposição ao metal, sendo que, entre estes, 83 são garimpeiros. Os pacientes com dosagens iguais ou acima de $10 \mathrm{ug} / \mathrm{l}$ e até 19 ug/l foram 16 (9,3\%), já assinalando grau moderado de exposição; 10 deles são garimpeiros.
Finalmente, em 9 pacientes $(5,2 \%)$, foram encontrados teores de $\mathrm{Hg}$ iguais ou maiores que 20 ug/l, considerados compatíveis com altos níveis de exposição ao metal. Destes últimos, 5 são garimpeiros.

Dentre esses 25 pacientes que apresentaram níveis de mercúrio iguais ou maiores que $10 \mathrm{ug} /$ 1,22 relataram sintomas compatíveis com quadro clínico de intoxicação mercurial crônica, enquanto que 3 não registraram queixa que pudesse ser associada a esse resultado.

TABELA 5. Dosimetria de Mercúrio Total em Urina, na População Amostral do Garimpo do Rato, Segundo Faixa Etária e Atividade Ocupacional. Itaituba, Pará

\begin{tabular}{|c|c|c|c|c|c|c|c|c|c|c|c|c|c|}
\hline \multirow[b]{3}{*}{ Faixa Etária } & \multicolumn{13}{|c|}{ Teor de Hg/Urina (ug/l) } \\
\hline & \multicolumn{2}{|c|}{$0-3$} & \multicolumn{2}{|c|}{$4-9$} & \multicolumn{2}{|c|}{$10-19$} & \multicolumn{2}{|c|}{$20-50$} & \multicolumn{2}{|c|}{+50} & \multicolumn{2}{|c|}{ População } & \multirow[b]{2}{*}{ Total } \\
\hline & G & NG & $\mathrm{G}$ & $\mathrm{NG}$ & G & NG & G & $\mathrm{NG}$ & G & NG & $\mathrm{G}$ & NG & \\
\hline $1-4$ & - & 2 & - & 2 & - & - & 1 & - & - & - & - & 5 & 5 \\
\hline $5-9$ & - & 2 & - & 1 & - & - & - & - & - & - & - & 3 & 3 \\
\hline $10-14$ & - & 5 & 1 & 1 & - & - & - & - & - & - & 1 & 6 & 7 \\
\hline $15-19$ & 4 & 1 & - & 1 & 1 & - & - & - & - & - & 5 & 2 & 7 \\
\hline $20-29$ & 29 & 15 & 12 & 14 & 5 & 1 & 1 & 1 & - & 1 & 47 & 32 & 79 \\
\hline $30-39$ & 1.5 & 7 & 8 & 9 & 3 & 2 & 3 & 2 & - & - & 28 & 21 & 49 \\
\hline $40-49$ & 4 & 2 & 5 & 1 & - & 1 & 1 & - & - & - & 10 & 4 & 14 \\
\hline $50-59$ & 1 & 2 & 2 & - & 1 & - & - & - & 1 & - & 5 & 2 & 7 \\
\hline Ignorada & 2 & - & - & - & - & - & - & - & - & - & 2 & - & 2 \\
\hline Total & 55 & 36 & 28 & 29 & 10 & 6 & 4 & 3 & 1 & 1 & 98 & 75 & 173 \\
\hline
\end{tabular}

$\mathrm{G}=$ garimpeiro

$\mathrm{NG}=$ não garimpeiro 


\section{Morbidade por Doença Sexualmente Transmissível}

Sífilis

Os resultados laboratoriais por faixa etária e sexo (Tabela 6) revelam 77 (42,0\%) indivíduos entre homens e mulheres reativos para o agente da sífilis, com predomínio de reação no sexo masculino (61 ou 33,0\%), em relação ao feminino (16 ou 9,0\%). Na primeira faixa de distribuição etária foi registrada uma criança do sexo masculino, com 9 anos, reagente para o agravo.

Ao considerar os dados apresentados na Tabela 6 de soropositividade nas categorias profissionais, observa-se maior prevalência entre os garimpeiros, 49/103 (48,0\%), seguido das prosti- tutas, 6/16 (38,0\%), mostrando uma alta freqüência de sífilis nestes grupos sociais.

\section{Hepatite B}

Os resultados da pesquisa laboratorial dos marcadores da Hepatite B estão contidos na Tabela 7. A prevalência para esse agravo é alta já na faixa etária de 0 a 10 anos - apesar do pequeno número de indivíduos entrevistados nesse intervalo - e sobe gradativamente com a idade até $100 \%$ nas faixas maiores do que 40 anos. A prevalência geral para o grupo ficou em $85,0 \%$. Dentre os 182 pacientes investigados, $11(6,0 \%)$ eram portadores do vírus (HBsAg positivos), sendo que a dosimetria de um deles acusava teores de mercúrio em 39,08 ug/l.

TABELA 6. Soroprevalência para Sífilis em Amostra da População do Garimpo do Rato, Segundo Faixa Etária e Sexo. Itaituba, Pará

\begin{tabular}{|c|c|c|c|c|c|c|}
\hline \multirow[b]{3}{*}{ Faixa Etária } & \multirow{3}{*}{ Total } & \multicolumn{4}{|c|}{ Sexo } & \multirow{3}{*}{ Prevalência \% } \\
\hline & & \multicolumn{2}{|c|}{ Masculino } & \multicolumn{2}{|c|}{ Feminino } & \\
\hline & & Reagente & Não Reagente & Reagente & Não Reagente & \\
\hline $0-10$ & 5 & 1 & - & - & 4 & 20,0 \\
\hline $11-20$ & 22 & 4 & 9 & 3 & 6 & 31,8 \\
\hline $21-30$ & 85 & 27 & 29 & 9 & 20 & 42,3 \\
\hline $31-40$ & 51 & 17 & 17 & 4 & 13 & 41,1 \\
\hline $41-50$ & 13 & 6 & 6 & - & 1 & 46,1 \\
\hline $51-60$ & 7 & 5 & 2 & - & - & 71,4 \\
\hline Ignorada & 2 & 1 & 1 & - & - & 50,0 \\
\hline Total & 185 & 61 & 64 & 16 & 44 & 41,6 \\
\hline
\end{tabular}

TABELA 7. Soroprevalência para Marcadores da Hepatite "B", na População do Garimpo do Rato, segundo Faixa Etária e Sexo. Itaituba, Pará

\begin{tabular}{|c|c|c|c|c|c|c|}
\hline \multirow[b]{3}{*}{ Faixa Etária } & \multicolumn{4}{|c|}{ Sexo } & \multirow{3}{*}{ Total } & \multirow[b]{3}{*}{ Prevalência \% } \\
\hline & \multicolumn{2}{|c|}{ Masculino } & \multicolumn{2}{|c|}{ Feminino } & & \\
\hline & Positivo & Negativo & Positivo & Negativo & & \\
\hline $0-10$ & - & 2 & 2 & 7 & 3 & 71,4 \\
\hline $11-20$ & 2 & 5 & 2 & 20 & 11 & 80,0 \\
\hline $21-30$ & 6 & 20 & 8 & 84 & 50 & 83,3 \\
\hline $31-40$ & 3 & 13 & 5 & 51 & 30 & 84,3 \\
\hline $41-50$ & - & 1 & - & 13 & 12 & 100,0 \\
\hline $51-60$ & - & - & - & 7 & 7 & 100.0 \\
\hline Total & 11 & 41 & 17 & 182 & 113 & 85,0 \\
\hline
\end{tabular}

Obs.: Dentre os pacientes da amostra, 11 eram HBsAg positivo. 


\section{Morbidade por Doenças do Aparelho Respiratório}

Dos entrevistados, $47 \%$ referiram queixas de "gripe" e, destes, 30\% assinalaram mais de 6 "episódios gripais" no último ano. A distribuição dos sintomas gripais referidos, destaca a tosse, mialgias (12\%), dispnéia (6\%), acompanhados de astenia (27\%), dor articular (14\%), e outros $(25 \%)$.

\section{DISCUSSÃO}

A escolha da amostra estatística adotada pelo Projeto Itaituba esteve condicionada a parâmetros correspondentes a certas características regionais que não podem ser ignoradas quando do planejamento de projetos na área de saúde: a falta de dados oficiais sobre as populações residentes na Amazônia, inclusive sobre saúde, as peculiaridades do processo de ocupação garimpeira e, finalmente, a situação de falência em que se encontra a prestação de serviços de saúde no País e particularmente na região amazônica.

A estimativa de 300 pessoas na área se mostrou realista. A decisão de trabalhar com a totalidade da população provou ser acertada, tendo em vista a enorme expectativa que desperta a presença de uma equipe de saúde entre uma população desassistida, tornando impossível recusar o atendimento médico, quando solicitado.

O caráter desordenado do processo de ocupação da terra por grupos garimpeiros desculparia, no primeiro momento, a ausência dos serviços de saúde e saneamento básicos nesses agrupamentos humanos. Entretanto, a realidade é que esses serviços são insuficientes ou ausentes em toda a região amazônica, mesmo em comunidades reconhecidas como cidades, salvo em certa medida, nas capitais. No garimpo do Rato, onde a exploração do ouro foi iniciada em 1963 na "corrutela do João Leite", 30 anos são passados e ainda não existe nenhum posto de saúde e as condições de saneamento são extremamente precárias.

A composição da população com predominância do sexo masculino é característica dos agrupamentos garimpeiros, todavia, o intervalo de variação das idades neste estudo, entre 6 meses e 59 anos, que não é habitual nesse tipo de população, deve-se ao fato de termos entre a amostra os moradores da "corrutela do João Leite", que já é um núcleo populacional razoavelmente estabelecido.

O baixo nível de instrução associado à precariedade dos serviços de saneamento e às condições de vida e de trabalho, concorre para agravar o quadro geral da saúde no local, onde as prováveis conseqüências da exposição ao $\mathrm{Hg}$ constituem apenas mais um componente, disputando espaço com as endemias, as doenças crônico-degenerativas e os acidentes de trabalho. Entre os dados gerais de morbidade destacam-se a presença da malária como agravo mais relatado, assim como a freqüência e variedade das parasitoses intestinais por indivíduo, ambas as situações concorrendo para o estado de anemia comum na população, além da alta prevalência da hepatite B observada laboratorialmente.

Sob o ponto de vista nutricional não parece haver carência alimentar grave de natureza protéico-calórica, apesar de o pescado não ser a principal fonte de proteínas, como acontece em grande parte das comunidades amazônicas (Giugliano et al., 1981, 1984). Entretanto, a dieta declarada pelos respondentes - da qual estão ausentes frutas e legumes - é pobre em vitaminas e sais minerais.

A população coexiste com a malária - que foi referida e constatada como agravo mais freqüente - com tranqüilidade e fatalismo, sem medo. É uma questão de tempo de verbo: a maioria "já teve" a doença, alguns "estão" no momento com ela e, certamente, mais dia menos dia, numa ocasião ou outra, todos eles "terão", ou voltarão a ter, o agravo. Essa situação é comum aos garimpos situados na Amazônia (Couto, 1988, 1991).

O resultado da leitura das lâminas revelando $35,0 \%$ de positividade entre os pacientes atendidos, sendo que dentre estes, $52,3 \%$ em indivíduos assintomáticos, chama a atenção para a dificuldade no controle da doença numa região em que a endemicidade e os procedimentos terapêuticos insuficientes já criaram um patamar de resistência que permite a situação encontrada (Silva et al., 1993). Em 6 desses indivíduos a dosimetria do mercúrio total, feita em urina, revelou 
estarem eles com teores de mercúrio acima de 20 ug/l, evidenciando mais uma vez a concomitância de agravos na mesma pessoa, possibilidade que deve ser levada em conta quando se tratar de populações na Amazônia.

A concentração de $\mathrm{Hg}$ medido na urina está associada ao horário em que a coleta é feita, ao intervalo de tempo entre essa coleta e a última exposição aos vapores do metal, à freqüência com que o indivíduo é exposto, e à cinética de excreção que varia de pessoa a pessoa (Piotrowski et al., 1975; Wallis \& Barber, 1982; Lie et al., 1982). Os espécimes de urina examinados pela pesquisa foram provenientes da primeira micção, tal como é preconizado; porém, não foram coletados dados que permitam associações individuais com o processo de queima do amálgama, que nos garimpos não é diário, e freqüentemente é realizado a céu aberto. Sob essas condições não se pode considerar que os níveis encontrados na urina correspondam necessariamente à concentração do metal na atmosfera do ambiente de trabalho (Yoshida \& Yamamura, 1982), ainda que permita a suposição de que os níveis individuais possam ser mais altos quando medidos próximo à queima do amálgama. Daí a necessidade de informações interdisciplinares, inclusive aquelas de natureza ambiental, para compor um quadro mais completo da questão da saúde humana em áreas garimpeiras (Santos, 1993).

Os parâmetros para o que é considerado normal encontrar de mercúrio metálico nas dosagens de urina humana são diferentes, dependendo das fontes consultadas. O valor de normalidade considerado pela OMS é de até 4 ug/l (Colacioppo, 1979; WHO, 1980), enquanto que para a Legislação da Segurança e Medicina do Trabalho - LSMD (1992) o valor da normalidade chega a $10 \mathrm{ug} / \mathrm{l}$, e para ambos $50 \mathrm{ug} / \mathrm{l}$ é o limite de tolerância para organismos humanos. Optamos, no momento, por trabalhar com a LSMD, até que seja conhecido um padrão que possa ser associado ao conceito de normalidade para o tipo de risco dos garimpos amazônicos.

Estudos feitos em situações de contaminação industrial do ambiente de trabalho por mercúrio, retratam situações de exposição ao metal muito diferentes daquela que acontece nos espaços abertos da Amazônia em conseqüência da atividade garimpeira, onde o risco de exposição, geralmente intermitente - e nem por isso menos prejudicial à saúde - precisa ser conhecido a partir de todas as diversas variáveis envolvidas, iniciando por um conhecimento melhor do meio ambiente e das populações envolvidas.

As determinações de mercúrio total feitas em 173 urinas pertencentes à amostra, revelaram 25 $(14,5 \%)$ indivíduos nos quais o resultado da dosagem ficou acima de $10 \mathrm{ug} / \mathrm{l}$. É interessante notar que alguns desses pacientes não apresentavam, no momento, sinais clínicos ou laboratoriais que pudessem estar associados à intoxicação pelo metal. Entretanto, apesar de não ter sido observada a intoxicação mercurial aguda do ponto de vista clínico, as análises laboratoriais detectaram concentrações anormais desse metal nos 25 pacientes citados, e diversos sintomas relatados coincidem com o quadro de manifestações crônicas.

Os sintomas clínicos compatíveis com o quadro de intoxicação mercurial crônica, nem sempre estiveram acompanhados por dosagens laboratoriais fora da normalidade, assim como alguns pacientes com teores de $\mathrm{Hg}$ acima de 10 ug/l não manifestaram sinais e sintomas de intoxicação. Essa observação chama a atenção para a necessidade do apoio laboratorial para os diagnósticos clínicos e para a necessidade do diagnóstico diferencial com outras doenças comuns na região, cujos sinais e sintomas possam coincidir com aqueles encontrados na intoxicação mercurial.

Vivendo em um ambiente no qual o Hg é usado diária e abundantemente, e queimado livremente, é certo que os habitantes da região num momento ou noutro, através de contacto direto com a forma líquida (Galvão \& Corey, 1987), inalando seus vapores ou - o que é mais grave ingerindo alimentos que possam estar contaminados com mercúrio orgânico, incorram no risco de intoxicação pelo metal.

A prevalência dos anticorpos contra o agente da sífilis, maior até mesmo do que é comum encontrar entre reclusos de penitenciárias (Andrade et al., 1989; Gomes, 1985), sugere tratar-se de uma área superendêmica, e reflete as condições de promiscuidade observadas entre a maior parte da população garimpeira.

A elevada prevalência dos marcadores da hepatite B, confirma as observações de Bensa- 
bath \& Dias (1983) e Bensabath \& Soares (1986), sobre a ocorrência da referida infecção em algumas áreas da Amazônia e especialmente nos grupos garimpeiros. Dos pacientes portadores do vírus (HBsAg positivos), em número de 11, quatro estavam com malária e um com a dosimetria para mercúrio em 39,08 ug/l no momento da investigação, chamando mais uma vez a atenção para a superposição de agravos, comum às condições de vida dessa área. É importante ratificar que além de ser uma doença de transmissão parenteral, com possibilidade de transmissão por contato com sangue e hemoderivados, a hepatite B é considerada importante doença sexualmente transmissível (Hadler \& Morgolis, 1989; Silva 1995).

Quanto às queixas clínicas registradas no decorrer do inquérito clínico-epidemiológico, e que podem estar associadas à intoxicação mercurial, mesmo quando apresentando correlação positiva, como é o caso da Tabela 4, temos a comentar que as mesmas são provenientes de indivíduos submetidos a condições de vida e de trabalho de tal modo agressivas, que fica difícil estabelecer com precisão as relações de causa e efeito. O máximo que podemos assegurar é que existe uma correlação positiva entre os sintomas referidos na tabela $4 \mathrm{e}$ o tempo de exposição do paciente ao metal.

Por outro lado, os médicos da rede de saúde, se a mesma está presente, carecem de treinamento especializado para reconhecer clinicamente um paciente de hidrargirismo, e como tratá-lo. Quando se leva em conta a natureza quelante das substâncias indicadas na terapêutica da intoxicação mercurial, emerge outra vertente do problema da contaminação que é a necessidade de monitoração laboratorial desses pacientes durante o tratamento, o que não existe na região (Joselow et al., 1972; ATSDR/EPA, 1989).

Sobre o desempenho destas populações, resta especular qual a eficiência de trabalho que se pode esperar de indivíduos anêmicos, constantes portadores de parasitos intestinais, submetidos a freqüentes crises de malária, já tendo passado por outras doenças, como por exemplo a hepatite $\mathrm{B}$ e sífilis, e ainda vivendo o alto risco de intoxicação por mercúrio.

A população estudada está submetida às mais variadas situações de agravo à saúde, inclusive aqueles em decorrência direta da ati- vidade garimpeira. A exposição ao mercúrio é um deles, o de consciência recente e o mais alarmante, à medida que se propaga contaminando o meio ambiente, não pode ser controlado apenas por recomendações de saúde individual ou coletiva, e cuja solução envolve os interesses econômicos de uma atividade produtiva de fiscalização difícil como é o processo garimpeiro. No entanto, os demais agravos constatados, que por incontáveis anos vêm debilitando a saúde do homem na Amazônia, e contribuindo para aumentar os índices de mortalidade, podem ser evitados, na maioria das ocasiões, por medidas de saneamento, prevenidos por vacinação, ou tratados, melhorando as condições de vida e de trabalho das populações residentes.

Concluindo, o presente trabalho ofereceu apenas uma visão geral das condições de saúde encontradas na comunidade garimpeira estudada. Impossível, sob pena de alongar demasiadamente o artigo, detalhar todos os diversos aspectos que foram pesquisados, e que serão objeto de publicações futuras. Mas essa visão geral atinge a finalidade de por em evidência a multicausalidade encontrada quando se precisa avaliar as condições de saúde na região amazônica, e que deve ser levada em consideração por ocasião do atendimento clínico e da administração terapêutica.

\section{AGRADECIMENTOS}

Aos moradores do garimpo do Rato, cuja colaboração nos permitiu desenvolver a pesquisa. Aos Srs. Ivo Lubrina de Castro, José Cavalcante (Paraíba) e Oliveira, pelo apoio operacional na área. À Unidade Mista da FNS em Itaituba na pessoa do Dr. Jorge da Silva Baena, pelo apoio laboratorial. Aos auxiliares técnicos de pesquisa do Instituto Evandro Chagas (IEC), Raimundo Otávio Paixão, Raimundo Pio Girard Martins, Airton da Graça Teixeira e Bernardo F. da Conceição, pela colaboração insubstituível. À Dra. Isabel Rodrigues do Serviço de Parasitologia do IEC, pela participação no trabalho de campo. Ao Dr. Alexandre Pessoa da Silva, coordenador do projeto Itaituba e consultor do LEHMA, pelo apoio ao laboratório de toxicologia. Aos Drs. 
Isabelle Roumieux e Luiz Augusto Galvão, da ECO/OPAS do México, e Maurício Andrade Peres, da UFRJ, pelo auxílio com o banco de dados. Ao geólogo da SEICOM, Artur Mascarenhas, pelas informações prestadas.

\section{RESUMO}

SANTOS, E. O.; LOUREIRO, E. C. B.; JESUS, I. M.; BRABO, E.; SILVA, R. S. U.; SOARES, M. C. P.; CÂMARA, V. M.; SOUZA, M. R. S. \& BRANCHES, F.

Diagnóstico das Condições de Saúde de uma Comunidade Garimpeira na Região do Rio Tapajós, Itaituba, Pará, Brasil, 1992. Cad. Saúde Públ., Rio de Janeiro, 11 (2): 212-225, abr/jun, 1995.

O objetivo do trabalho foi conhecer as condições de vida e de saúde de uma comunidade garimpeira e, particularmente, a superposição da contaminação Mércurial com outros agravos. Foi realizada no garimpo do igarapé do Rato, na bacia hidrográfica do rio Tapajós, Itaituba-Pará. A população abrangeu 223 indivíduos aos quais foi aplicado um questionário epidemiológico, e feito atendimento médico e coleta de espécimes biológicos. Na coproscopia, 96,1\% indivíduos estavam parasitados e $66,4 \%$ anêmicos. A prevalência para o vírus da hepatite $\mathrm{B}$ ficou em $85,0 \% ; 11(6,0 \%)$ eram portadores do vírus. Dentre os pacientes investigados para malária, $35,0 \%$ (65) estavam com a doença e desses $52,3 \%$ (34) eram pacientes assintomáticos. A prevalência para sífilis foi de $41.6 \%$. As dosagens de mercúrio em urina de 173 indivíduos revelaram $16(9,2 \%)$ pacientes com dosagens maiores do que $10 \mathrm{ug} / \mathrm{l}$ e até $19 \mathrm{ug} / \mathrm{l}$, enquanto que 9 pacientes $(5,2 \%)$ do total investigado revelaram teores acima de $20 \mathrm{ug} / \mathrm{l}$. O estudo revela indivíduos com superposição de agravos, sem diagnóstico adequado por falta de atendimento médico, praticando a automedicação, e, agora, vivendo a ameaça da intoxicação por mercúrio.

Concluindo, o presente trabalho ofereceu apenas uma visão geral das condições de saúde encontradas na comunidade garimpeira estudada. Impossível, sob pena de alongar demasiadamente o artigo, detalhar todos os diversos aspectos que foram pesquisados, e que serão objeto de publicações futuras. Entretanto, essa visão geral atinge a finalidade de por em evidência a multicausalidade encontrada quando se precisa avaliar as condições de saúde na região amazônica, e que deve ser levada em consideração por ocasião do atendimento clínico e da administração terapêutica.

Palavras-Chave: Saúde Ambiental; Saúde do Trabalhador; Garimpo de Ouro; Mercúrio

\section{REFERÊNCIAS BIBLIOGRÁFICAS}

ATSDR (Agency for Toxic Substances and Disease Registry U.S. Public Health Service) \& EPA (Environmental Protection Agency U.S.), 1989. Toxicological Profile for Mercury. Washington, DC: Clement Associates.

ANDRADE, A. L. S. S.; MARTELLI, C. M. T.; SOUSA, L. C. S.; SOUSA, M. A. E. \& ZICKER, F., 1989. Soroprevalência e fatores de risco para sífilis em população carcerária de Goiás. Revista do Instituto de Medicina Tropical de São Paulo, 31: 177-182.

BENSABATH, G. \& DIAS, L. B., 1983. Hepatite de Lábrea (febre de Lábrea) e outras hepatites em Sena Madureira, Acre e Boca do Acre, Amazonas, Brasil. Revista do Instituto de Medicina Tropical de São Paulo, 25: 182-194.

BENSABATH, G. \& SOARES, M. C. P., 1986. Hepatites por vírus. In: Instituto Evandro Chagas; 50 Anos de Contribuição às Ciências Biológicas e à Medicina Tropical (Instituto Evandro Chagas, org.), vol. 2, pp. 560-570, Belém: Fundação Serviços de Saúde Pública.

BRABO, E. S., 1992. Contaminação por Mercúrio dos Rios Crepori e Marupá - Bacia do Tapajós. Tese de Mestrado, Belém: Universidade Federal do Pará.

CÂMARA, V. M. \& COREY, G., 1992. Epidemiologia e Meio Ambiente. O Caso dos Garimpos de Ouro no Brasil. Metepec, México: Centro Panamericano de Ecologia Humana e Saúde/ECO/OPS.

CAMPOS, R. C. \& PIVETTA, F., 1993. Análise de mercúrio em sangue, urina e cabelo. In: Mercúrio em Garimpos de Ouro (V. M. Câmara, org.), pp. 65-77, Metepec, México: Centro Panamericano de Ecologia Humana e Saúde/ECO/Organização Panamericana da Saúde. (Série Vigilância, 12) 
COLACIOPPO, S., 1979. Avaliação do risco de intoxicação profissional por vapores de mercúrio. Revista Brasileira de Saúde Ocupacional, 7: 4661.

CONAMA (Conselho Nacional do Meio Ambiente), 1986. Resolução № 20 de 18 de junho de 1986. Brasília, DF: Ministério do Desenvolvimento Urbano e Meio Ambiente. (Mimeo.)

COUTO, R. C. S., 1988. Intoxicação mercurial: resultados preliminares em duas áreas garimpeiras no Estado do Pará. Pará Desenvolvimento, IDESP, 23: 63-67.

1991. Buscando Ouro, Perdendo Saúde: Um Estudo Sôbre as Condições de Saúde no Garimpo do Cumarú - Pará. Tese de Mestrado, Rio de Janeiro: Escola Nacional de Saúde Pública, Fundação Oswaldo Cruz.

DNPM (Departamento Nacional de Produção Mineral), 1990. Cadastro de Garimpos. Brasília, DF: Ministério da Infra-Estrutura.

FÉLIX, J. T., 1987. Análise da produção de ouro no Brasil. Brasil Mineral: 48: 90-96.

FERNANDES, R. C. F. \& PORTELA, I. C. M. H., 1991. Recursos Minerais da Amazônia - Alguns Dados Sobre Situação e Perspectivas. Rio de Janeiro: Centro de Tecnologia Mineral. (Série Estudos e Documentos, 14)

GALVÃO, L. A. C. \& COREY, G. 1987. Mercurio. Edo. de Mexico: Centro Panamericano de Ecología Humana y Salud, OPSIOMS. (Serie Vigilancia, 7)

GIUGLIANO, R.; GIUGLIANO, L. \& SHRIMPTON, R., 1981. Estudos nutricionais das populações rurais da Amazônia. I. Várzea do Rio Solimões. Acta Amazônia, 11: 773-788.

GIUGLIANO, R.; SHRIMPTON, R.; MARINHO, H. A. \& GiUGliANO, L., 1984. Estudos nutricionais das populações rurais da Amazônia. II. Rio Negro. Acta Amazônia, 14: 427-449.

GOMES, S., 1985. Incidência de reações sorológicas positivas para sífilis na população carcerária de Niterói. Arquivos Brasileiros de Medicina, 54: 275-278.

HACON, S. P., 1991. Mercury Contamination in Brazil, with Emphasis on Human Exposure to Mercury in Amazonian Region. Rio de Janeiro: WHO/UNEP.

HADLER, S. C. \& MORGOLIS, H. S., 1989. Viral hepatitis. In: Viral Infections of Humans, Epidemiology and Control, (A. S. Evans, ed.), pp. 351-391, Nova York: Plenum Medical Book.

JOSELOW, M. M.; LOURIA, M. D. \& BROWDER, A. A., 1972. Mercurialism: environmental and occupational aspects. Annais of Internal Medicine, 76: 119-130.

LSMD (Legislação da Segurança e Medicina do Trabalho), 1992. Legislação da Segurança e Medicina do Trabalho. $2^{\underline{a}}$ ed., São Paulo: Editora LTR.

LIE, A.; GUNDERSEN, N. \& KORSAARD, K. J., 1982. Mercury in urine - sex, age and differences in a reference population. Scandnavia Journal Work Environment Health, 8: 129-133.

MARTINELLI, L. A.; FERREIRA, J. R.; FOSBERG, B. R. \& VICTORIA, R. L., 1988. Mercury contamination in the Amazon: A gold rush consequence. AMBIO, 17: 252-254.

PFEIFFER, W. C.; LACERDA, L. D.; MALM, O.; SOUZA, C. M. M.; SILVEIRA, E. G. \& BASTOS, W. R., 1989. Mercury concentrations in inland waters of Rondonia, Amazon, Brazil. Science Total Environmental, 87/88: 233-240.

PIOTROWSKI, J. K.; TROJANOWSKA, B. \& MOGILNICKA, E., 1975. Cinetic of excretion and urine mercury variability in workers exposed to mercury vapor. Archives Occupacional Environment Health, 35: 245-256.

RAMOS, J. F. F. \& COSTA, M. Q., 1991. Distribuição de mercúrio em dois garimpos do Estado do Pará. In: Riscos e Conseqüências do Uso do Mercúrio (S. Hacon; L. D. Lacerda; W, C. Pfeiffer \& D. Carvalho, eds.), pp. 70-79, Rio de Janeiro: Finep/ $\mathrm{CNPq} / \mathrm{Ibama} / \mathrm{MS}$.

SANTOS, E. O., 1993. Garimpagem e saúde. In: Conseqüências da garimpagem no âmbito social e ambiental da Amazônia (A. Mathis \& R. Rehaag, eds.), pp. 36-72, Belém: FASE/ Buntistift e Katalyse.

SEICOM (Secretaria de Indústria, Comércio e Mineração/Programa de Controle Ambiental da Garimpagem do Rio Tapajós), 1992. Estudos dos Impactos Ambientais Decorrentes do Extrativismo Mineral e Poluição Mercurial do Tapajós. Belém: Seicom.

SILVA, P. R. N., 1995. Hepatite Aguda por viris A, B, C, D, E, F e G. In: Doenças Sexualmente Transmissíveis (M. R. P. Passos, ed.), 4를. ed., Rio de Janeiro: Cultura Médica.

SILVA, R. S. U.; SANTOS, E. C. O.f LOUREIRO, E. C. B.; JESUS, I. M.; CÂMARA, V. M.; BRABO, E. S.; FERNANDES, A. S. \& SOUZA,J. M., 1993. Malária Assintomática - Uma das Causas da Perpetuação da Cadeia da Transmissão da Malária. IV Reunião dos Pesquisadores em Malária, Resumos, Manaus: Governo do Estado do Amazonas. (Mimeo.) 
TODD \& SANSORD E DAVIDSOHN, 1982. Diagnósticos Clínicos e Conduta Terapêutica por Exames Laboratoriais. Ed. Manole.

YOSHIDA, M. \& YAMAMURA, Y., 1982. Elemental mercury in urine from workers exposed to mercury vapor. Archives Occupacional Environment Health, 51: 99-104.
WALLIS, G. \& BARBER, T., 1982. Variability in urine mercury excretion. Journal of Occupacional Medicine, 24: 290-295. 